Appl i cat i on of the Repul si ve- Type Magnet i c Bearing for Nanuf act uring Mcromass Measur ement Bal ance Equi pment

\begin{tabular}{|l|l|}
\hline 著者 & $\begin{array}{l}\text { Hussi en Al aa A., Yamada Sot oshi, I wahar a } \\
\text { Nasay oshi, Okada Tont ada, Ohj i Takahi sa }\end{array}$ \\
\hline $\begin{array}{l}\text { j our nal or } \\
\text { publ i cat i on ti t l e }\end{array}$ & I EEE Tr ansact i ons on Nagnet i cs \\
\hline vol une & 41 \\
\hline nunber & 10 \\
\hline page r ange & $3802-3804$ \\
\hline year & $2005-10-01$ \\
\hline URL & ht t p: //hdl . handl e. net /2297/11881 \\
\hline
\end{tabular}




\title{
Application of the Repulsive-Type Magnetic Bearing for Manufacturing Micromass Measurement Balance Equipment
}

\author{
Alaa A. Hussien ${ }^{1}$, Sotoshi Yamada ${ }^{1}$, Member, IEEE, Masayoshi Iwahara ${ }^{2}$, Associate Member, IEEE, \\ Tomotada Okada ${ }^{1}$, and Takahisa Ohji ${ }^{3}$, Member, IEEE \\ ${ }^{1}$ Institute of Nature and Environmental Technology, Kanazawa University, Kanazawa, Ishikawa 920-8667, Japan \\ ${ }^{2}$ Graduate School of Nature Science and Technology, Kanazawa University, Kanazawa, Ishikawa 920-1192, Japan \\ ${ }^{3}$ Department of Electrical and Electronic Engineering, Faculty of Engineering, Toyama University, Toyama 930-8555, Japan
}

\begin{abstract}
Many applications of the single-axis controlled repulsive-type magnetic bearing have been proposed earlier. However, both passive and active magnetic bearings are employed in most of such systems. In this paper, a new permanent magnet (PM) repulsive-type micromass measurement system is developed. The repulsive forces of the PM section are used effectively to keep the radial direction stable where the electromagnets, control circuits, and peripheral devices needed for controlling the unstable direction are simplified. The configuration, control design, and operating characteristics of the proposed system have been presented.
\end{abstract}

Index Terms-Balance system, control design, magnetic bearing, mass measurement, permanent magnet repulsive force.

\section{INTRODUCTION}

$\mathbf{O}$ $\mathrm{NE}$ of the simplest methods of magnetic levitation technology is the repulsive-type magnetic bearing using permanent magnets (PMs). In fact, it is well known that at least one direction should be actively controlled in order to achieve a stable noncontact levitation condition in the magnetostatic field [1]. The single-axis controlled repulsive-type magnetic bearing system is studied by many researchers where it has the feature of reduction of peripheral devices and displacement sensors for active control [2]-[4]. It also has a soft and constant stiffness to the passive control axes, hence, in recent years, many applications employing this system, such as turbomolecular pumps, conveyor systems, and high-speed polygon scanner motors, have been proposed [5].

In this paper, a mass measurement balance system employing passive magnetic levitation units is proposed; this system is used for measuring the small masses with satisfactory measurement characteristics. The principle of measuring depends upon transferring the mass of the sample to the current of a voice coil motor (VCM) device, and the incremental change of this current denotes the mass value. The advantage of the proposed system over the conventional ones is that the distance between mass holder and the other system parts is big enough, hence, it can be applied for thermobalance to detect the difference between the mass of a reference sample and a measuring object while they are subjected to atmospheric condition changes, such as heating or pressurization. This technique is widely used for differential thermal analysis (DTA) and thermogravimetric analysis (TGA). Moreover, the measurement characteristics of the proposed system are considered satisfactory, at least, for the present time compared to its simple and low cost design.

Digital Object Identifier 10.1109/TMAG.2005.854929

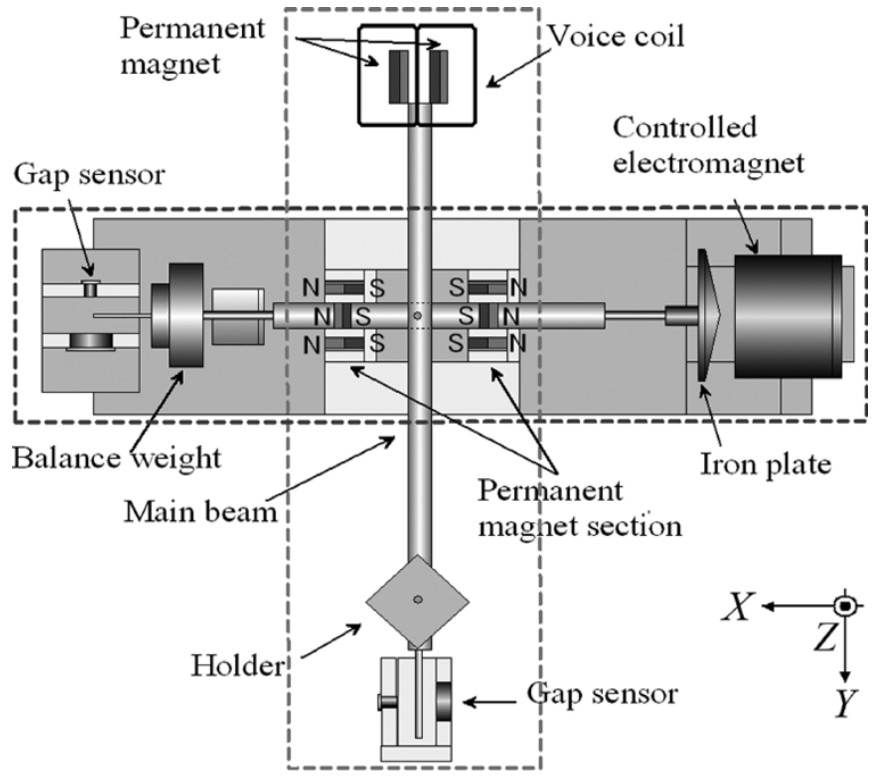

Fig. 1. Top view of the proposed balance equipment. Two passive magnetic bearing units are put for noncontact levitation along $z$-direction.

\section{SYSTEM CONFIGURATION AND BASIC CHARACTERISTICS}

The configuration of the proposed balance system is shown in Fig. 1. It consists of two main parts, namely: 1) the axial shaft part; and 2) the balance beam part. In the shaft part, two PM units are installed around the center point for levitating the system stably along the radial direction ( $z$-direction) where the configuration of the PM unit is shown in Fig. 2. The model of PM is analyzed using the finite element method (FEM), where the calculated and measured repulsive force characteristics are shown in Fig. 3. The shaft is unstable along the axial direction ( $x$-direction) so an electromagnet is designed and placed beside an iron plate that is fixed at one end of the shaft. The attraction force of the electromagnet is needed for stabilizing the shaft. The information of axial position of the shaft is detected by a 


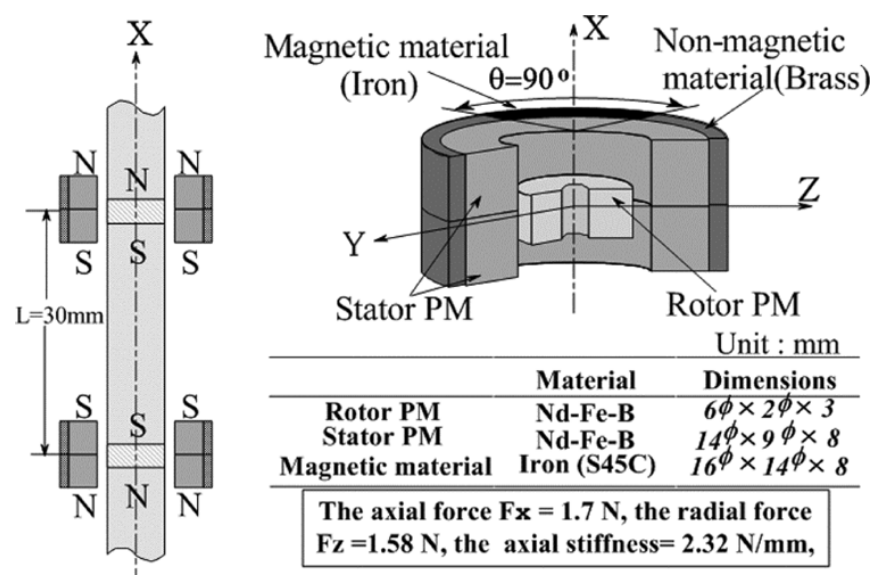

Fig. 2. PM unit configuration. Both stator and rotor are cylindrical and magnetic material covers a part of the stator is attached to make it easy to control the axial direction.

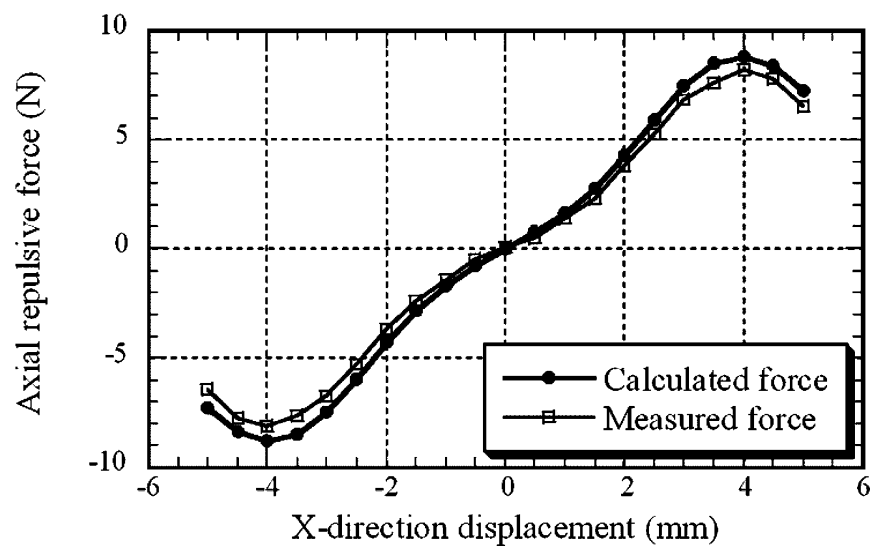

Fig. 3. Characteristics of the PM repulsive force along the axial direction. The calculated and measured forces are given to show the data matching.

gap sensor installed at the other end of the shaft; this information is supplied to an axial position controller to modulate the electromagnet current and, consequently, the force to achieve stable noncontact levitation.

The balance beam is attached to the shaft where the sample holder is placed at one side of the beam. A VCM is designed for controlling the beam position. It consists of an iron yoke fixed at the other side of the beam, two pieces of PMs attached to the yoke, and a coil. When the sample is dropped into the holder, the beam position is changed; this change is detected by a gap sensor located beside the holder. The sensor signal is transferred to the position controller that modulates the coil current for returning the beam to its initial equilibrium position, and the sample mass is transferred to the voice coil current.

\section{System Modeling AND CONTROL DeSIGN}

The models of both axial shaft and balance beam are shown in Fig. 4. The shaft model is mathematically described by (1) and (2) where $m$ is the mass of the levitating object and $x$ is the axial displacement. $R, L, i$, and $e$ are the electromagnet resistance, inductance, current, and voltage, respectively. The electromagnet attractive force $\left(F_{m}(x, i)\right)$ and the axial repulsive force of PM

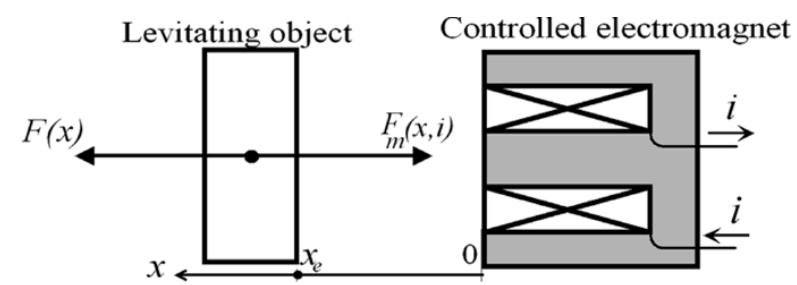

(a)

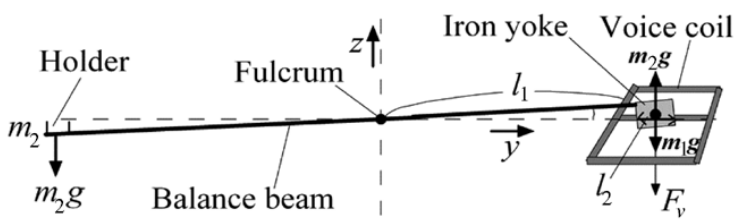

(b)

Fig. 4. Dynamic system model for derivation of the mathematical equations. (a) Shaft model. (b) Beam model.

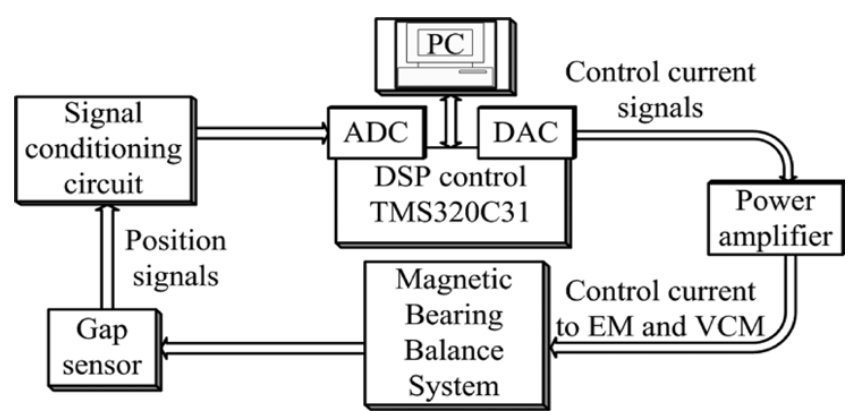

Fig. 5. Block diagram of the controlled system. The position of both shaft and beam is detected by a photosensor. The steady electromagnet current is $0.45 \mathrm{~A}$ and the voice coil steady current is $0.1 \mathrm{~A}$.

at the linear region around the operating point $(F(x))$ are given in (3) and (4), respectively, where $K, a$, and $b$ are constants

$$
\begin{aligned}
m \ddot{x} & =F(x)-F_{m}(x, i) \\
R i+L \dot{i} & =e \\
F_{m}(x, i) & =k\left(\frac{i}{x}\right)^{2} \\
F(x) & =a x+b .
\end{aligned}
$$

The beam model is described in (5) and (6) where $m_{1}$ and $m_{2}$ are the mass of the yoke and the measured sample, respectively, $z$ is the vertical displacement of the yoke, and $g$ is the gravity. The VCM force $\left(F_{v}\right)$ is given in (7) where $B$ is the flux density inside the gap of the yoke. $N, l_{2}$, and $i_{v}$ are the coil turns, length, and current, respectively

$$
\begin{aligned}
m_{1} \ddot{z} & =m_{2} g-m_{1} g-F_{v} \\
m_{1} \ddot{y} & =0 \\
F_{v} & =i_{v} B l_{2} N .
\end{aligned}
$$

The state space representation of the linearized models could be easily obtained; however, the control systems that control the axial direction displacement of the shaft and vertical direction displacement of the beam are designed using the linear quadratic optimal control technique. The controllers are simulated by the aid of Matlab Simulink and configured around a digital signal processor (TMS320C31-DSP) where the block diagram of the controlled system is shown in Fig. 5. The steady levitation characteristics for both shaft and beam are measured, and the vibration is kept within $10 \mu \mathrm{m}$ for the shaft and within $25 \mu \mathrm{m}$ for the 


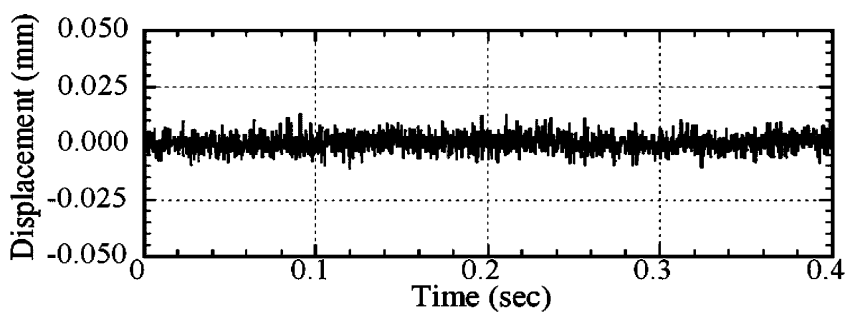

Fig. 6. Steady levitation characteristics of beam control. The range of beam vibration is kept within $25 \mu \mathrm{m}$ under the steady levitation condition.

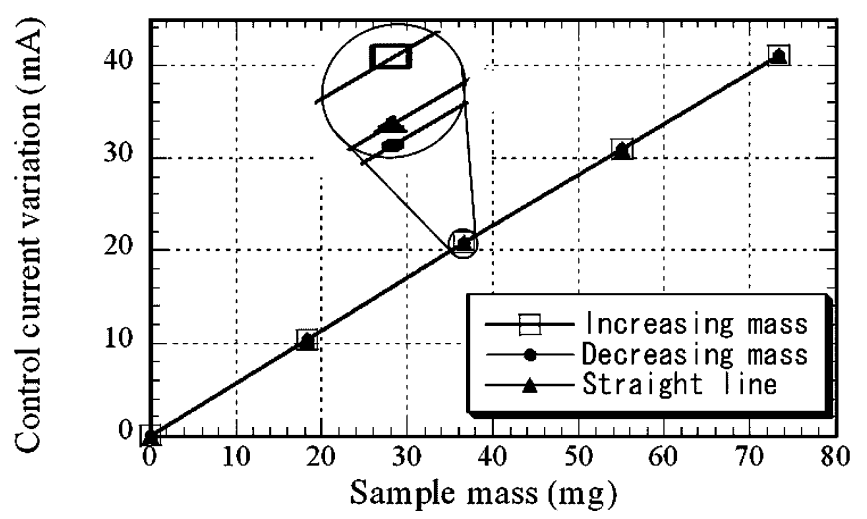

Fig. 7. VCM current variation versus sample mass characteristic. The straight line passing through zero is provided to show the close-up of the data.

beam. Fig. 6 shows the beam displacement along the $z$-direction versus time characteristic.

\section{EXPERIMENTAL RESULTS}

After the system has successfully stabilized and the steadystate levitation condition is achieved, the experiments are carried out on the system. Several samples of nominally equal mass are prepared for testing the control current linearity and the ability of the system to have a consistent sensitivity throughout the measuring range. These samples are put into the mass holder gradually and removed gradually while the VCM control current is measured; this current characteristic is shown in Fig. 7. The nonlinearity error of the current is $0.2 \%$ of the full-scale current; it indicates that the system sensitivity $(0.584 \mathrm{~mA} / \mathrm{mg})$ is nearly consistent over the measuring range. The difference in the VCM current reading at the same mass value where that value is approached by increasing and decreasing the mass, respectively, is $4 \mu \mathrm{A}$; this difference is termed as hysteresis error and is equivalent to a mass error of $7 \mu \mathrm{g}$.

To prove that the system measures the correct sample mass, ten standard samples in the range from 0 to $100 \mathrm{mg}$ are measured by our system. The actual mass of these samples is checked by the Mettler Toledo balance. The accuracy error and standard deviation of the system could be obtained. Fig. 8 shows that the absolute value of the maximum accuracy error is $0.2 \mathrm{mg}$ and the standard deviation is $0.1 \mathrm{mg}$. Fig. 9 shows the samples' measured mass versus samples' actual mass characteristic.

The estimated equation and correlation coefficient of this characteristic prove a perfect linearity. The minimum mass detected by the equipment is $30 \mu \mathrm{g}$, whereas the maximum

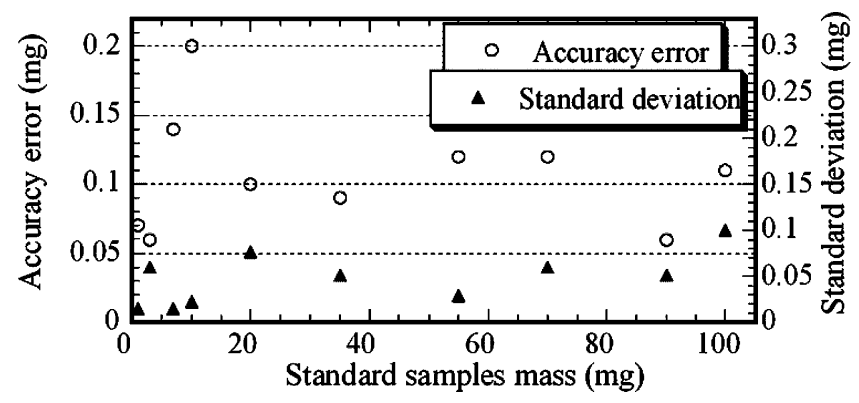

Fig. 8. Accuracy error absolute value and standard deviation characteristics. The standard samples are measured by the proposed system to show the reliability of the system for independent measurement.

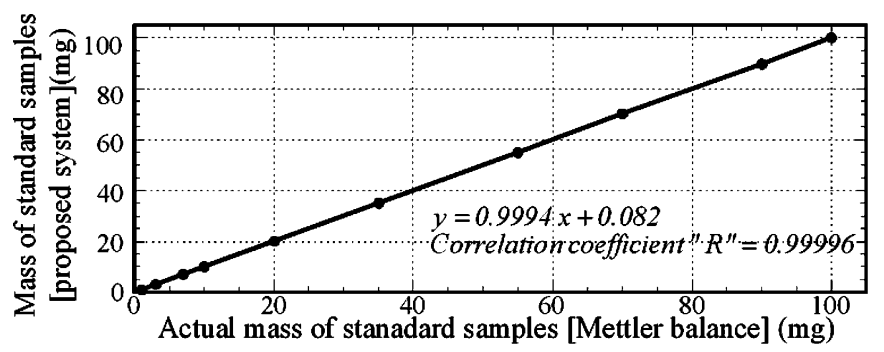

Fig. 9. Measured mass versus actual mass characteristics. The equation and correlation coefficient of the characteristics are estimated.

mass is $100 \mathrm{mg}$; this indicates that the system resolution is $0.03 \%$.

\section{CONCLUSION}

The repulsive-type magnetic bearing is applied for manufacturing a balance system that is considered as the primary design for thermobalance systems. This system is used for measuring the sample mass by transferring the value of that mass to a voice coil current. The structure of the proposed system and basic operating characteristics were presented in this paper. In addition, the models and control design of the system were included. The experiments were carried out on the system, and it is proven that the system measures the correct sample mass. The absolute value of the accuracy error was $0.2 \mathrm{mg}$ and the standard deviation was $0.1 \mathrm{mg}$. The minimum detected mass was $30 \mu \mathrm{g}$, whereas the full capacity is $100 \mathrm{mg}$ (resolution $=0.03 \%$ ).

\section{REFERENCES}

[1] J. P. Yonnet, "Permanent magnetic bearing and coupling," IEEE Trans. Magn., vol. MAG-17, no. 1, pp. 1169-1173, Jan. 1981.

[2] T. Ohji, S. C. Mukhopadhyay, M. Iwahara, and S. Yamada, "Permanent magnet bearing for horizontal- and vertical-shaft machines-A comparative study," J. Appl. Phys., vol. 85, no. 8, pp. 4648-4650, Apr. 1999.

[3] S. C. Mukhopadhyay, T. Ohji, M. Iwahara, and S. Yamada, "Modeling and control of a new horizontal shaft hybrid type magnetic bearing," IEEE Trans. Ind. Electron., vol. 47, no. 1, pp. 100-108, Feb. 2000.

[4] T. Ohji, S. Yamada, and M. Iwahara, "Methods of adjusting the force characteristics between the rotor and stator permanent magnets to develop a small-sized repulsive type magnetic bearing" (in Japanese), $J$. Magn. Soc. Jpn., vol. 25, no. 4-2, 2001.

[5] T. Ohji, S. Ichiyama, K. Amei, M. Sakai, and S. Yamada, "Conveyance test by oscillation and rotation to permanent magnet repulsive-type conveyor," IEEE Trans. Magn., vol. 40, no. 4, pp. 3057-3059, Jul. 2004.

Manuscript received February 4, 2005 